\title{
A VARIATIONAL METHOD FOR SOLVING QUASILINEAR ELLIPTIC SYSTEMS INVOLVING SYMMETRIC MULTI-POLAR POTENTIALS
}

\author{
Ali Jabar Rashidi, Mohsen SheKarbaigi
}

Abstract. In this paper, a system of quasilinear elliptic equations is investigated, which involves multiple critical Hardy-Sobolev exponents and symmetric multi-polar potentials. By employing the variational methods and analytic techniques, the relevant best constants are studied and the existence of $\left(\mathbb{Z}_{k} \times \mathbb{S O}(N-2)\right)^{2}$-invariant solutions to the system is established.

Mathematics subject classification (2010): 35A15, 35B33, 35J70.

Keywords and phrases: Variational method, quasilinear elliptic system, critical Hardy-Sobolev exponent.

\section{REFERENCES}

[1] B. Abdellaoui, V. Felli, I. Peral, Existence and nonexistence for quasilinear equations involving the p-Laplacian, Boll. Unione Mat. Ital. Sez. B. 9, (2006), 445-484.

[2] H. Attouch, G. Buttazzo, G. Michaille, Variational analysis in sobolev and BV spaces, The Society for Industrial and Applied Mathematics, 3600 University City Science Center, Philadelphia, PA. 19104-2688.

[3] L. Caffarelli, R. Kohn, L. Nirenberg, First order interpolation inequality with weights, Compos. Math. 53, (1984), 259-275.

[4] D. CAO, P. HAN, Solutions to critical elliptic equations with multi-singular inverse square potentials, J. Differential Equations. 224, (2006), 332-372.

[5] D. CAO, S. PENG, A note on the sign-changing solutions to elliptic problems with critical Sobolev and Hardy terms, J. Differential Equations. 177, (2001), 494-522.

[6] F. CAtrina, Z. WANG, On the Caffarelli-Kohn-Nirenberg inequalities: Sharp constants, existence (and nonexistence) and symmetry of external functions, Comm. Pure Appl. Math. 54, (2001), 229-257.

[7] K. CHOU, C. CHu, On the best constant for a weighted Sobolev-Hardy inequality, J. London Math. Soc. 333, (2007), 889-903.

[8] Y. Deng, Q. GaO, D. Zhang, Nodal solutions for Laplace equations with critical Sobolev and Hardy exponents on $\mathbb{R}^{N}$, Discrete Contin. Dyn. Syst. 14, (2006), 707-719.

[9] V. Felli, S. TERRACINI, Elliptic equations with multi-singular inverse-square potentials and critical nonlinearity, Comm. Partial Differential Equations. 31, (2006), 469-495.

[10] A. Ferrero, F. Gazzola, Existence of solutions for singular critical growth semilinear elliptic equations, J. Differential Equations. 177, (2001), 494-522.

[11] J. P. Garcia Azorero, I. Peral Alonso, Multiplicity of solutions for elliptic problems with critical exponent or with a nonsymmetric term, Trans. Amer. Math. Soc. 323:2, (1991), 877-895.

[12] N. Ghoussoub, C. YuAn, Multiple solutions for quasilinear PDEs involving the critical Sobolev and Hardy exponents, Trans. Amer. Math. Soc. 352, (2000), 5703-5743.

[13] G. Hardy, J. Littlewood, G. Pólya, Inequalities, Cambridge University Press, Cambridge, UK, (1934).

[14] P. Han, Quasilinear elliptic problems with critical exponents and Hardy terms, Nonlinear Anal. 61, (2005), 735-758.

[15] Y. HUANG, D. KANG, On the singular elliptic systems involving multiple critical Sobolev exponents, Nonlinear Anal. 74, (2011), 400-412. 
[16] E. JANnELLI, The role played by space dimension in elliptic critical problems, J. Differential Equations. 156, (1999), 407-426.

[17] D. KAng, On the quasilinear elliptic problems with critical Sobolev-Hardy exponents and Hardy terms, Nonlinear Anal. 68, (2008), 1973-1985.

[18] D. KANG, Quasilinear elliptic problems involving multiple critical Hardy-Sobolev exponents, Computers and Mathematics with Applications 57, (2009), 107-116.

[19] D. KANG, Concentration compactness principles for the systems of critical elliptic equations, Differ Equ Appls. 4, (2012), 435-444.

[20] P. L. LIONS, The concentration compactness principle in the calculus of variations, the limit case (I), Rev Mat Iberoamericana. 1, (1985), 145-201.

[21] P. L. LiOns, The concentration compactness principle in the calculus of variations, the limit case (II), Rev Mat Iberoamericana. 1, (1985), 45-121.

[22] R. PALAIs, The principle of symmetric criticality, Comm Math Phys. 69, (1979), 19-30.

[23] D. SMETS, Nonlinear Schrödinger equations with Hardy potential and critical nonlinearities, Trans. Amer. Math. Soc. 357, (2005), 2909-2938.

[24] S. TERRACINI, On positive solutions to a class of equations with a singular coefficient and critical exponent, Adv. Differential Equations. 2, (1996), 241-264.

[25] J. VAZQUEZ, A strong maximum principle for some quasilinear elliptic equations, Appl Math Optim. 12, (1984), 191-202.

[26] M. WiLleM, Analyse fonctionnelle élémentaire, Paris: Cassini Éditeurs. (2003). 